\title{
Anthropological Documentation and Personal Information Management
}

\section{Documentation in the framework of the Mediafrica Project}

Mediafrica is a development cooperation project sponsored by the Finnish International Development Agency, FINNIDA. It is housed at the Global Music Centre in Helsinki. The theme of the project is "Music and Modern Media in Nordic / SADCC Cultural Exchange". SADCC stands for Southern African Development Coordination Conference, a community of ten states suffering from dependence of the Republic of South Africa. It is the purpose of the project to increase circulation of music material in the region and between the north and the south by tapping the potential inherent music workers and in new media technology. Until now special attention has been given to promotion in radio music production.

Although the aims are purely practical the project methodology is scientific in its essence, trying to combine research and development in an optimum manner. The project work is conceived as being based on the participatory research approach, which ensures a high level dialectic relation between the subjects and the objects, ultimately aiming at transforming all participants into concious subjects in the development process. 
Theoretically the work falls back on achievements in ethnomusicology, which is the study of music in its cultural context - in a sense the anthropological study of music. Thus data gathering techniques are methodologically speaking the same as in cultural anthropology while the special essence of music presents a number of problems, which have to be tackled in ways unique to this field of study.

The participatory research approach avoids the artificial opposition between desk versus field work by making both the researcher and the informant active agents in the problem solving process. The office moves out to the studied cultural setting, while on the other hand the office itself becomes an important location for actions of the empirical study.

One of the implications of this kind of approach is that the work has to be carefully documented so that it can be properly analyzed. Instead of under-estimating the administrative aspects of the undertaking, these are themselves made into a productive factor. The interactive formulation of problems, the selection of strategies - as well as the other links in the chain of knowledge production - are themselves culturally influenced considerations which can bring a lot of understanding if properly analyzed. Much more trivial is the fact that routines of purchase, correspondence, phone calls can communicate significant information which should be recorded and utilized.

The necessity of effective means of documentation is accentuated in the Mediafrica project where a lot of long distance communication takes place. Every piece of information lost could be needed in the process of future work. The ability to correctly understand the cultural processes involved in the development process is determined by access to information on quickly changing situations.

The project workers are naturally also carrying out fact finding and data gathering tours in the southern target area, which are extremely expensive. It is of vital importance that these tours should produce a maximum of output. There is no place for cultural development and research tourism in this context. On the contrary, situations should be utilized to produce a maximum of information and follow-up.

It is in this context that computerized procedures of information management come into the picture. Little analysis has been made on the effect of choices of computer type and program environment on the use of computers in the research process. The Mediafrica project has opted for PC compatible machines and the Windows environment. 
The machine choice was made for historical reasons: Once starting to invest in one line products, it has so far been difficult to shift to another. Second to this comes the low price level of PC compatibles and their accessories, as well as the existing wide knowledge base on this equipment. Windows was selected since it was an easy to learn and easy to teach Graphical User Interface, well suited to the relevant tasks. Its flexibility in program development is specially appealing. Although Windows is considered a difficult programming environment, the related tools are cheap and efficient.

These selections are of course not meant to be a statement in favour of the machine and environment in question. It is in fact quite easy to prove that both the Apple and the Next machines with their Apple-DOS and Unix environments, offer a number of decisive advantages in the music oriented work which Mediafrica is performing.

Cultural researchers have not been quick in beginning to utilize computers as an aid in actual research. Mostly the use has been limited to the preparation of manuscripts. In the case of archives, computers have also been seen as a new way of storing and organizing existing data bases. It was after the mid 80's when the computer entered into the very data collection process, when the first experiments were made in writing field diaries on lap tops.

This is why there is very little experience to fall back on, at the outset of a computer-aided cultural research and development project. Commercial program development has so far also had rather little to contribute. Project management as well as documentation are two areas where a number products of interest have emerged. Unfortunately these programs have not yet reached at a stage where they would be well suited for research.

My interest lies in a genre of programs which is less ambitious in its goals than the two categories mentioned. Personal Information Management refers to computer-aid in the daily tasks of office oriented work. How could I do my daily work in a more planned and organized manner? Could I schedule my limited time resource better and push through the too various tasks which are hanging over me? What is the best way of keeping order among the numerous contacts which I have to develop to achieve my task? These are a couple of the problems that Personal Information Management tries to solve by supplying a set of computer based tools. 
Personal Information Management packages - or PIMs as the they are called in the computer jargon - such as Way You Work, Your Way, On Time and Agenda - often include a calendar, an address book, a cardfile, a to-do list, sometimes even a simple document storage and retrieval system, automatic dialler or a E-Mail application. The present generation of this program category still suffers from a number of defects: severe limitations on number of addresses which can be stored, cryptic file names, excessive size etc. Low prices (150 - 400 US\$) ensure though that in the near future a range of highly useful systems are available at an affordable price.

Mediafrica started before this new wave of programs had reached Finland. Documentation needs were catered for by using the base utilities coming with Windows. They provided a good base line for the documentation needs of the project. Then the Windows version of Microsoft's word processor arrived. Having quite a different price level it was less appealing. The highly developed document template capabilities including WordBASIC programming ability were the reasons to give it a try. These qualities gave the kick off to documentation development ending up in series of small programs producing bit by bit full scale solutions to the problems formulated. These are the aspects of development work for the Mediafrica Personal Information Management system that I am going to describe in the next sections.

\section{Potentials of the Windows GUI utilities for ethnomusicological documentation}

I will demostrate this by illustrating the use of some of the Windows utilities as tools in the Mediafrica office.

A program manager is one of the most basic aids in organizing daily work. Apart from giving easy access to the most often used programs through their icons, it can also offer access to central files by symbolic presentations of the data files. Progman, the Windows Program Manager, organizes the Mediafrica desk top into three fields with the project files in the right-most section. Here all the vital information of the personal information management system is readily available with a couple of mouse button clicks: the daily calendar, the task list, the local and international address cardfiles, the diary and the phone log as well as the phone dialling utility: 


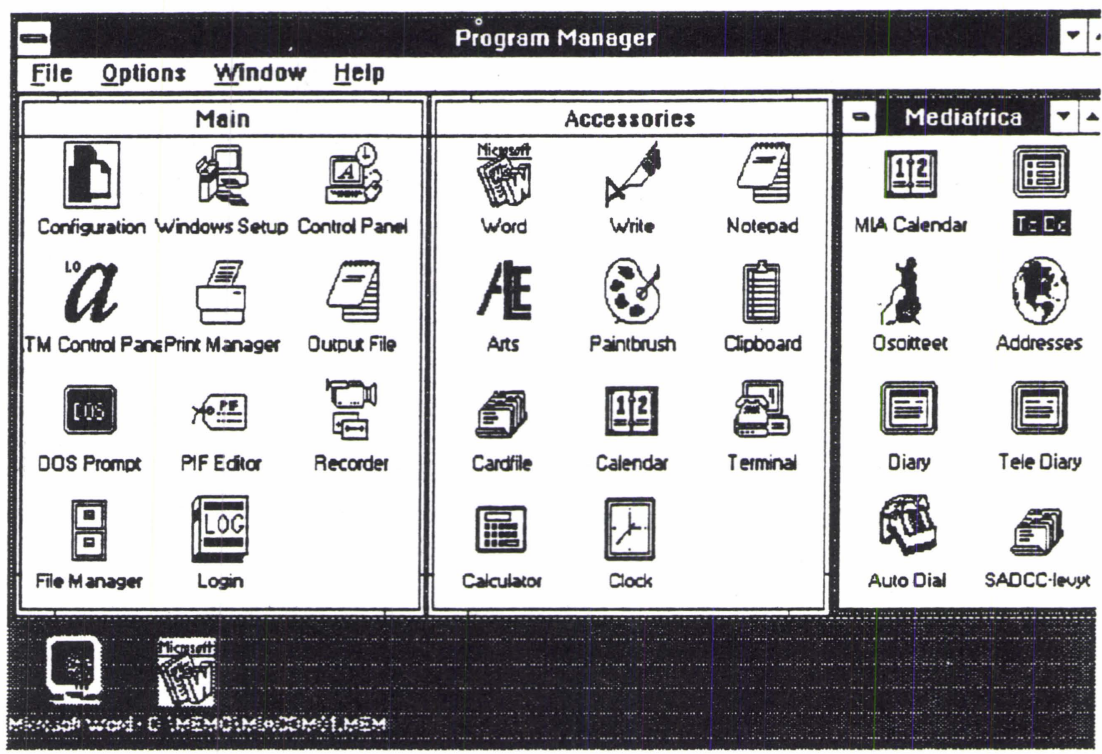

The project documents are organized in a two level tree structure where the name of the worker comes first, and secondly the document type. These document designations are mostly the direct name of the document type such as MEMO, DIARY, LETTER, FAX, MESSAGE etc. Documents are stored with a type reference which are the first letters of the document type: [filename].MEM for a MEMO, [filename].LET for a LETTER. The File Manager gives easy access to these documents. As correspondence is coded with a letter string (consisting most often the default, which is the six first letters of the first name) all documents sent to a particular receiver can be easily retrieved. This is done with the Search command of the program. This is what part of the Fax directory looks like:

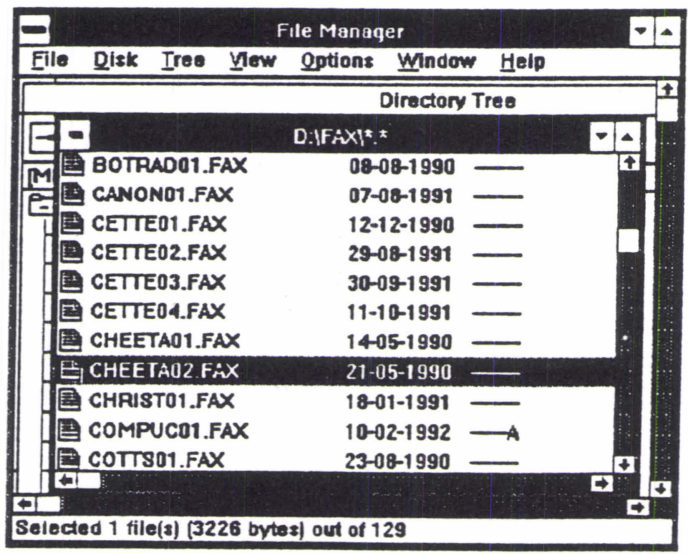


Some of the utilities do not require long description. Calc, which combines a simple arithmetic calculator with a scientific one, has been helpful in quick calculation of expenses, as well as in translating foreign prices into Finnish currency. Write is a proper word processing program, which can give a quick remedy to lack of a professional program. It has been helpful in producing neat copies of important files on the road in a hotel room. Even a fancy invitation or program can be made with one of the Windows stock fonts. Terminal has grown into something more than a simple way of communicating with bulletin boards systems. It has provided us with a means of exchanging both text and program files. Paintbrush is helpful in sketching objects, musical instruments, recording sites and maps of band member positions.

Cardfile is a simple database, which is presented as set of cards to the user. This program has been used in Mediafrica for a big number of different duties. Catalogues of slides, gramophone records, equipment to be purchased and other similar lists are easy to maintain. The most basic use is that of an address database. The program is rather well suited for this task. Through a menu selection the program can retrieve a phone number on the card and dial it if the computer has a modem. An address catalogue can also be produced - with little effort even as a text file. Being a Nordic project the address databases also include Scandinavian characters. Although Windows in itself gives strong language specific support, Cardfile does not make use of it and becomes confused in trying to sort the records. A total lack of a backup facility is another serious shortcoming of the program. Saving multiple instances of the same file in wrong order crashes in fact the file and makes it unreadable: A serious shortcoming indeed for a database program.

Calendar can perform a number of useful tasks. An appointment list is one of the items in the Mediafrica Program Manager window. Important appointments can be marked. A special command produces an alarm when the relevant task is due. At the bottom of each day there is a small scratch pad. This has been useful in drawing up rough preliminary work schedules and programs for visitors. The calendar has a published file format, which is also the case with Cardfile. Therefore these data files can be used by other programs, which will be demonstrated below when a calendar file is transformed into a dialling log. 
As the calendar can be written into a text file, work schedules can form the nucleus for a list of contents of a detailed diary. This is what has been done during Mediafrica SADCC surveys. The title page of the report is followed by the Calendar schedule which gives easy access to diary items. Word for Windows Goto buttons pointing at the relevant diary items could transform the list of contents into a hypermedial tool.

Below is a Calendar presentation of the first real working day of the Taarab Survey of February-March 1991. It includes a lot of activities which would have been difficult to keep track without this simple aid:

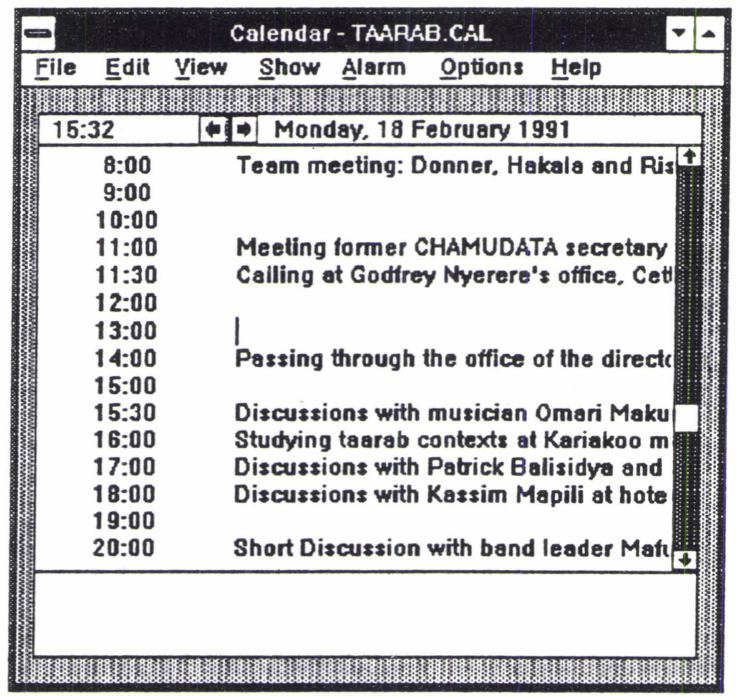

The calendar line can accommodate altogether 80 characters, although only the first of them are visible in window.

Most of the calendar items are parallelled by diary notes. These were simply made with the Notepad utility, the minimum word processor accompanying the Windows package. The program has the handy ability of inserting the current date - an important property in this context (newer versions supply even the time). At home these diary items were printed into a text file and then read into the real word processor. After a minimum of editing and lay-out the first version of the report was ready in its binder. This is part of one of the items of the example day: 


\section{Time: $\quad$ 18-02-1991, 15:30 \\ Place: $\quad$ Dar es Salaam, the out-door cafeteria at Hotel New Africa \\ Informants: Omari Makuka, Simo Rista \\ Author: PHD.}

1. At a glass of much too sweet Milinda I gave to Simo my version of taarab and its cultural contexts, focusing my presentation on visual and photographic aspects.

2. We then decided to go out and survey photography conditions. The idea was just to stroll around and get an idea of contexts and light environments. While Simo was fetching a small camera and some film the musician Omari Makuka of M.K. Group entered the cafeteria. I invited him to sit at my table and he gladly accepted.

3. I told Makuka that I had just given Simo my conception of the taarab, a presentation based on my personal experience. - What would he answer if asked by an outsider: 'Taarab ni kitu gani?' [What is Taarab?] He answered by giving a list of different kinds of songs: The first and foremost variety is "mahaba", then "nyimbo za serikali" [government songs], "za nchi zinaingizwa" [they are introducing songs about the country]. -I urged him to explain the word 'mahaba'? "Hasa ni mapenzi" [It's love affairs].

5. Then he went on explaining the poetical techniques, especially the use of metaphors. He took an example, a song describing a cup, "kikombe". Kikombe "ni mtu, kiungo maalum" [The cup is a person, a special creation]. Then he explained that the singer would sing: "Kila mtu anatumia hiki kikombe". Kwa hiyo "mimi siwezi" [Every person wants to use this cup. Therefore I can't.]

The program manager still includes one program of interest. The log icon represents a utility which helps in supplying multi-user ability to the information management system. When activated or whenever Windows is restarted (even after screen blanking) the following dialogue box displaying the users is popping up. The user selection steers the process of reading in correct information on the user's directories and default information management files into the general Windows initialisation file, were it is easily accessible to applications:

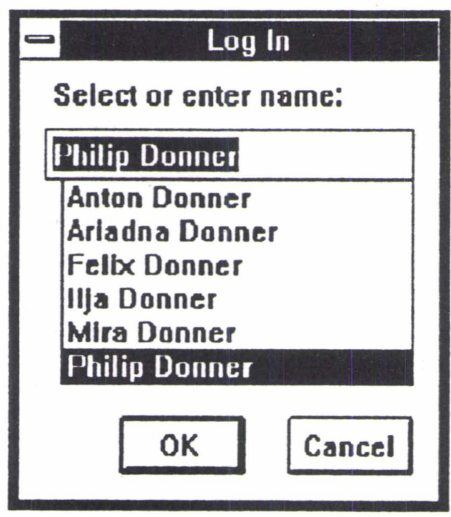




\section{An Auto-Dialler Supporting Systematic Collection of Data through Phone Calls}

Although seldom approved of as a valid technique in anthropological data collection, brief telephone conversations have proved to be a highly productive instrument in a problem oriented research setting. I am referring to a number of research efforts close to me: starting with the music study of Tuusula commune, a descriptive overview of the music of the Swedish-speaking minority in Finland and a survey of Finnish music policy.

Productivity can be accelerated through suitable audio recording equipment connected to the phone and systematical documentation of the calls. This is possible by manual methods, but computer-aid can add to the efficiency, especially through automatic dialling, logging and report generation.

In trying to improve the throughput of telephone communications within the Mediafrica project, the first solution was to rely on the auto dialling capabilities of Cardfile, one of the standard Windows utilities. Sensing that the limitations and problems of this program could be avoided, an attempt was first made to steer Cardfile through macro procedures written in the WordBASIC language, which is part of Microsoft Word for Windows. A high level of external control was achieved, but in order to further add in flexibility and speed, autonomous utilities were written in the $C$ language.

Instead of describing the development cycle step by step, we'll plunge straight into the resultant application called Dial, and its support libraries. While scanning through basic functions of the program a couple of aspects demonstrating the capabilities of the Windows GUI (Graphical User Interface) will be brought up.

After activation of the Dial icon - not so surprisingly a visual abstraction of a telephone - a modeless dialogue box shows up:

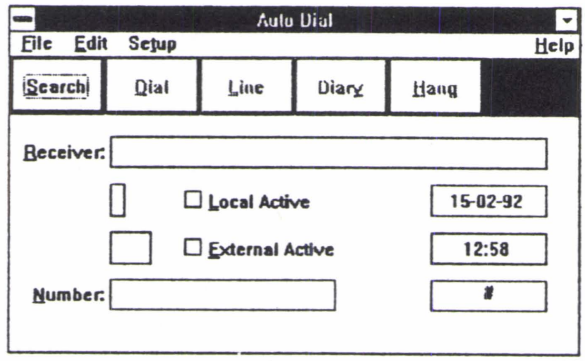


Although highly complex in its appearance (although visually not as fancy as some of the commercial programs available to-day) this front end can be produced rather quickly by utilizing one of the many dialogue box generators now available.

The front end utilizes a number of pre-programmed resources: pop-up menus, push buttons, check boxes, static and editable text windows - to mention a few. Through these resources and brief sequences of Windows commands a high degree of functionality can be achieved.

It is no big deal to prevent the user from dialling as long as no phone number has been entered. Similarly, diary reporting and phone hang up are enabled only after the operator has gone on-line. The check boxes automatically configure for house, local and external calls. Checking the boxes and linking this with activation of prefix numbers are again trivial programming tasks. All the methods mentioned can in fact be automatically produced by utilizing one of the qualified program generators available these days.

Higher level of functionality such as use of pass words, configuration of the modem, a link to the address cardfiles, diary and log file set up, automatic reporting and logging, were all added through a more extensive programming effort. Without entering into all the details of the program, let us have a look at the implementation of the interface to Cardfile data files.

After the operator has pushed the search button the program blocks further activation by greying and thus disabling the search button. Then another modal dialogue box pops up:

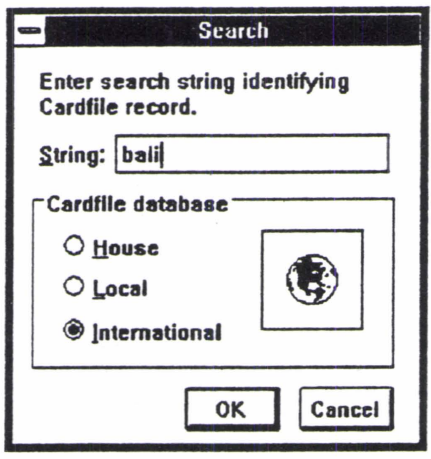

Here the user interacts with the program by (a) entering a string of characters, (b) selecting the relevant address data file and finally (c) 
confirming the input. What is special here is first the icon field which presents a globe which is a resource owned by another program, namely the control panel. In the Windows environment icons and other resources can be shared between different programs. Multiple use of the same capability goes even further than this. The Search box is actually not part of the Dial program, but it is itself also a shared resource, being part of a so-called dynamic link library (DLL), a module of program code, which is loaded whenever a program needs its services. Thus the Search box can be used not only by the Dial program but by any other Windows program which needs its services.

The auto dialler is organized around the idea of records into two or three categories, in order to filter unwanted matches and to speed up the up-dates of the data base. In case the dialling system operates under any kind of telephone centre the option of an in house call is available to the user. These are made without the internal prefix. Selection of the local option will activate the prefix if thus configurated, while the international option will supply the external call prefix if detecting a ' + ' sign in the beginning of the number.

In the Mediafrica information management scheme the search box and the following select card box are also used in locating addresses, fax numbers, file codes etc:

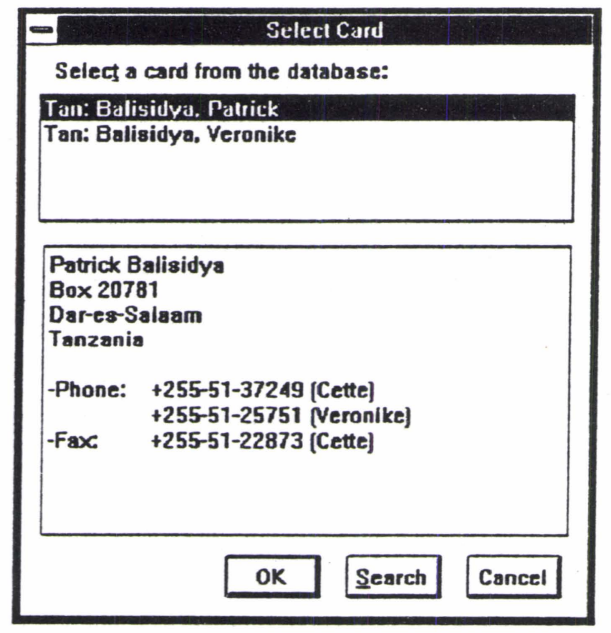

This is a browser into Cardfile compatible databases. After entering at least a sub-string of the indexed information (in this case 'bali'), the index records conforming to the entered character string are loaded into the list box. The DLL function amplifies Cardfile in two ways. 
Instead of having to perform a number of consecutive search commands, the relevant card can be immediately displayed by selecting the record in the list box. The list box makes use of Windows' support of culture specific information. Sorting of the strings in the list box is done according to the language selected in the Control panel. This browser could with a small programming effort be added to Cardfile itself, by adding a menu selection linking the extension to all instances of the program.

The phone number is extracted with the help of the '-Phone:' parameter designation. The hyphen is not obligatory, but ensures correct identification of the parameter name, since the Cardfile display does not differentiate linefeeds from a wrapped line. The two carriage returns after the address intentional. Apart from enhancing readability of the card, they also give a way of extracting the address from the card. By using simple country coding - 'Tan:' for Tanzania, 'Zim:' for Zimbabwe - related records are grouped together in the database. If the user would need to browse through all the records from Botswana, this would be possible by entering 'bot:' into the search box and then selecting the international database option.

After the card selection the receiver and telephone numbers are loaded into the relevant fields. The dial button is now highlighted and the thick edge signals that it is the default selection. The External check box is selected and the configured number ' 990 ' as well as the accompanying text are highlighted.

\begin{tabular}{|c|c|c|c|c|}
\hline \multirow{2}{*}{\multicolumn{5}{|c|}{ Eile Edit Sefup }} \\
\hline & & & & \\
\hline Search & $\overline{\mathrm{Di}}$ & !nine & Dianx & Hang \\
\hline \multicolumn{5}{|c|}{ Beceiver: Patrick Balisidya } \\
\hline & $\Pi$ & \multicolumn{2}{|c|}{$\square$ Local Active } & 15-02-92 \\
\hline External: & 990 & \multicolumn{2}{|c|}{ 区xternal Active } & 13:15 \\
\hline Number: & $255-$ & -37249 & & 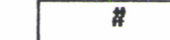 \\
\hline
\end{tabular}

Now we are ready to fire an automatic pulse or tone coded dialling sequence by pushing the button. 
At this point one might ask the trivial question: Why is all this fuss needed in order to dial a number? Apart from taking care of the retrieval of numerous phone numbers, the program gives a higher level of exactness in entering the data. Assuming that the data record is correct the dialler will reproduce it in a fool-proof way any number of times. External calls, especially those made to the SADCC area are often hard to get through. The program supports a better throughput by duplicating the repeat button of advanced phones.

Other advantages include automatic reporting and logging capabilities. The program supports a simple report template which can be modified by the user. Data on the receiver, number, date and time of the phone call are entered into the 's\%' marked fields. Also note the initials of the author which are initialized during the log in process. This kind of data merge into character block is again a function which does not require programming efforts. It is supported directly in Windows. Here is the template and the beginning of our sample report:
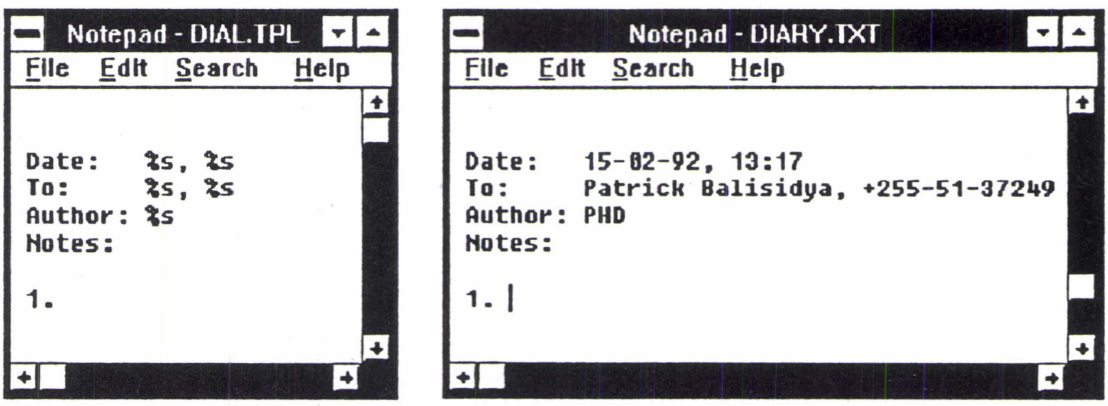

Another DLL, which again can be called from any other Windows program, will produce a Calendar record in the log file configurated through one of the Setup menu selections. We will use another day in the Mediafrica log, which is a bit more instructive. Instead of looking at the data through the Calendar window let's have a look at a print out of the days records:

8:39 Call to Jan Verweijnen, $787449 \rightarrow$ TELELOG3.DLA

8:50 Call to Risto Rännilä, $13415509 \rightarrow$ TELELOG3.DLA

18:52 Call to Johanna Jauho, 1461741 -> TELELOG4.DLA 
This day three of the out-going calls were recorded according to the Calendar utility's file format. In addition to the information reproduced in the phone diary, the log also indicates the file were the report can be found.

One more aspect deserves to be described. What is the magic behind automatic location of the address data bases. In addition to a general initialisation file called WIN.INI, Windows supports the use of initialisation files for individual programs. The program file DIAL.EXE and the template file DIAL.TPL are accompanied by DIAL.INI which stores most of the configuration information. Though it can be modified by any Windows editor such as Notepad it is recommendable to let the program supply the values of the parameters. This ensures the validity of the data. Anyhow it might be interesting to have a look at DIAL.INI:

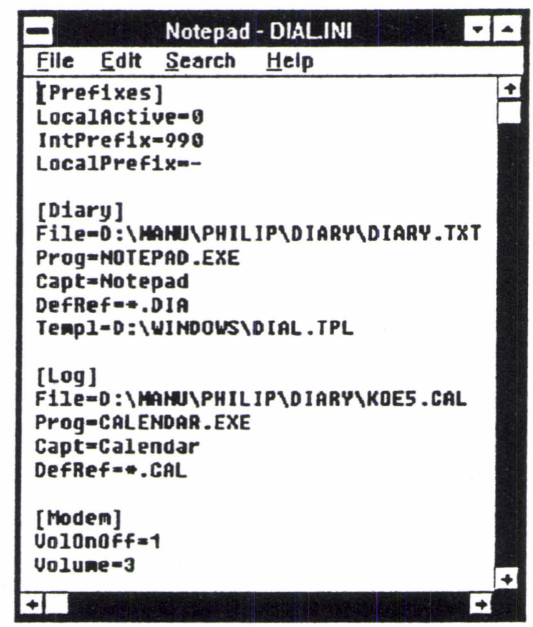

Each section of the file have linkage to the selections under the Setup menu. The default settings of the prefix windows are under the [Prefix] item. The file parameter indicates where the logs and diaries are to be found. [Modem] lists some of the properties which can be programmed in Hayes compatible modems. Others are included in the WIN:INI. That is also where the address files are stored since the parameters are used by multiple programs of the information management system. 